\title{
On the Yield Stress of Sheared Coagulated Suspensions
}

\author{
Motoyoshi KOBAYASHI ${ }^{1}$, Setsuo $\mathrm{OOI}^{2}$, Yasuhisa ADACHI ${ }^{3}$ \\ ${ }^{1}$ Member of JSCE, Dr. (Agr. Sci.), Laboratory of Water Environmental Engineering, University of Tokyo \\ (Yayoi 1-1-1, Bunkyo-ku, Tokyo 113-8657, Japan) \\ ${ }^{2}$ Dr. of Agr. Sci., National Institute for Rural Engineering (Kannondai 2-1-2, Tsukuba 305-8609, Japan) \\ ${ }^{3}$ Dr. of Agr. Sci., Institute of Agricultural \& Forest Engineering, University of Tsukuba
}

(Tennodai 1-1-1, Tsukuba 305-8572, Japan)

\begin{abstract}
In the present study, we derived a model of yield stress of coagulated suspensions by developing our viscosity model previously proposed. Evaluated values of yield stress by the present model showed good agreement with the experimental results by Buscall et al., who measured yield stress of polystyrene latex suspension coagulated with $0.1 \mathrm{M} \mathrm{BaCl}_{2}$ solution, where the electrical repulsive force between particles is negligible. From the analysis, it is expected that flocs become compact in higher values of volume fraction and prolonged shearing makes flocs compact.
\end{abstract}

Key Words: rheology, flocculation, colloid, floc, fractal dimension

\section{INTRODUCTION}

In the field of hydraulic engineering, to predict and control the movement of coagulated suspensions (cohesive sediments, fluid mud, and slurry) are desired ${ }^{1-3)}$. Generally, coagulated suspensions show non-Newtonian fluid behaviors such as shear-thinning (decrease of viscosity with increasing shear rate) and yield stress (stress at that suspension can start to flow). These rheological behaviors are strongly related to the particle-particle interaction in suspensions. That is, colloidal particles contained in a coagulated suspension coagulate and form flocs (aggregates). Formation of flocs gives rise to the increase of the effective volume fraction of particles and the increase of the viscosity of suspension because the liquid cannot easily flow through inside flocs. Thus, to predict the start and stop of motion of cohesive sediment, it is important to clarify the relationship between rheological properties of coagulated suspensions and physical properties of flocs in relation to particle-particle interaction.

Recently, we derived a theoretical model describing the shear viscosity of coagulated suspensions by taking into account the size and fractal structure of flocs in suspensions subjected to shear ${ }^{4)}$. This model well describes the flow curves of coagulated polystyrene latex (PSL) suspensions (Fig. 1). In the present study, we propose a model of yield stress by developing our model for viscosity. Evaluated values by the proposed model showed good agreement with the experimental data by Buscall et al. ${ }^{5)}$, who measured the yield stress of PSL aqueous dispersions coagulated with $0.1 \mathrm{M}$ $\mathrm{BaCl}_{2}$ as functions of volume fraction of particles and diameter of PSL particles.

\section{DERIVATION OF YIELD STRESS MODEL}

Shear stress $(\tau)$ is given by a product of suspension viscosity $\left(\mu_{s}\right)$ and shear rate $(G)$. Fig. 1 shows the log-log plot of $\tau\left(=\mu_{s} G\right)$ vs. $G$ evaluated

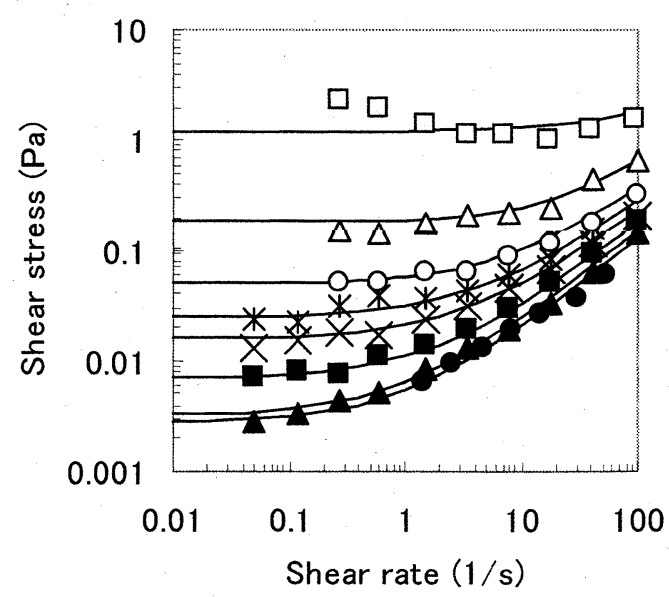

Fig. 1 Shear stress $(\tau)$ vs. shear rate(G) for PSL suspensions taken from ref. (4). Lines and symbols denote evaluated values and experimental data, respectively. Volume fraction of particles are 0.322 , $0.216,0.15,0.117,0.077,0.045,0.031$, and 0.014 from upper curve to lower. 
by our viscosity model ${ }^{4)}$. As shown in this figure, $\tau$ reaches to a certain constant value in the regime of lower values of $G$. These constant stress values are considered to be yield stresses $\left(\tau_{y}\right)$. Next, keeping this in mind, we will consider flocs in the suspension at the condition where shear stress is constant.

In a coagulated suspension, the effective volume fraction of flocs $\left(\phi_{f}\right)$ is given by the following equation ${ }^{4)}$ :

$$
\phi_{f}=\left(\frac{d_{f}}{d_{0}}\right)^{3-D} \phi_{0}
$$

where $d_{f}, d_{0}$, and $\phi_{0}$ denote diameter of a floc, diameter of primary particles, and volume fraction of primary particles, respectively. $D$ is so-called fractal dimension and is in the range of $1 \leq D \leq 3$. It should be kept in mind that flocs with higher $D$ have compact structure. Diameter of flocs $\left(d_{f}\right)$ in a concentrated suspension subjected to shear stress $\tau$ $\left(=\mu_{s} G\right)$ is expressed by the following equation ${ }^{4}$ :

$$
d_{f}=\left(\frac{d_{0} A}{9 \pi \tau h^{2}}\right)^{\frac{1}{2}}
$$

where $A$ is Hamaker constant, $h$ is the minimum separation distance between particle surfaces. Eq. (2) supposes that van der Waals attractive force is dominant for interparticle forces because we assume the case where the electrical repulsive force between particles is negligible. By using Eqs. (1) and (2), one can calculate $\phi_{f}$. In Fig. 2, calculated values of $\phi_{f}$ with the same parameters as used in the evaluation of viscosity ${ }^{4)}$ shown in Fig. 1 are plotted against $G$. This figure indicates that the value of $\phi_{f}$ reaches to the value of volume fraction at the condition of cubic packing $\left(\phi_{m}(=\pi / 6=0.52)\right)$ as $G$ decreases. At the condition of cubic packing, it is considered that suspensions are not able to flow smoothly and continuously ${ }^{4,10}$.

From the behaviors of coagulated suspensions as shown in Figs. 1 and 2, we can deduce the following condition for yield stress:

$$
\tau \rightarrow \tau_{y} \text { for }=\phi_{f} \rightarrow \phi_{m}(=0.52)
$$

Finally, combining Eqs. (1-3), one can obtain the following equation to calculate yield stress:

$$
\tau_{y}=\frac{A}{9 \pi d_{0} h^{2}}\left(\frac{\phi_{0}}{0.52}\right)^{\frac{2}{3-D}}
$$

\section{EVALUATION OF YIELD STRESS COMPARED WITH EXPERIMENTAL RESULTS}

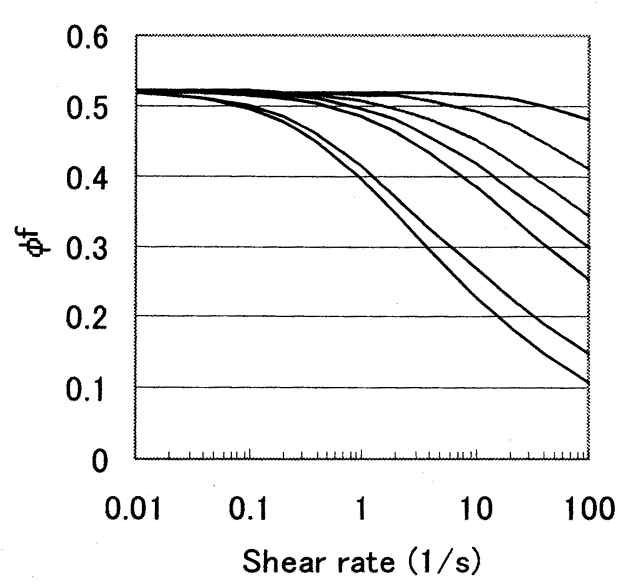

Fig. 2 Evaluated effective volume fraction of flocs $\left(\phi_{\mathrm{f}}\right)$ vs. shear rate $(\mathrm{G})$. Values of volume fraction of particles are $0.322,0.216,0.15,0.117,0.077,0.045$, 0.031 , and 0.014 from upper curve to lower.

In this chapter, we evaluate $\tau_{y}$ by Eq. (4) proposed above to compare the evaluated values with experimental data by Buscall et al.. Evaluations of $\tau_{y}$ were performed for four different diameters of primary particles $\left(d_{0}=0.488 \times 10^{-6} \mathrm{~m}\right.$, $d_{0}=0.966 \times 10^{-6} \mathrm{~m}, \quad d_{0}=2.621 \times 10^{-6} \mathrm{~m}$, and $\left.d_{0}=3.400 \times 10^{-6} \mathrm{~m}\right)$, various $D(2.0-2.5)$, and $\phi_{0}(0.05-0.26)$ according to the experimental condition of Buscall et al. ${ }^{5)}$. The same value of $A / h^{2}=1 \times 10^{-3} \mathrm{~J} / \mathrm{m}^{2}\left(A=10^{-21} \mathrm{~J}, h=10^{-9} \mathrm{~m}\right)$ as used in the previous study ${ }^{4)}$ for the evaluation of the viscosity of coagulated PSL suspension was adopted. Also, it is expected that the value of $D$ increase with increasing $\phi_{0}{ }^{4,6,7)}$.

\section{RESULTS AND DISCUSSION}

Fig. 3 shows the relationships between yield stress $\left(\tau_{y}\right)$ and volume fraction of particles $\left(\phi_{0}\right)$. In this figure, symbols and lines denote experimental data by Buscall et al. ${ }^{5)}$ and evaluated values by the present model, respectively. In Fig. 3, symbols with "Before" stands for the data obtained by the first measurement after suspensions were set up in a viscoelastic analyzer. "After" stands for the data obtained from the suspension after prolonged shearing. According to Buscall et al., the values of yield stress of "after" samples are smaller than those of "before" samples.

As shown in Fig. 3, the experimental values of yield stress obey the one of the lines drawn by the present model. From the comparison of evaluated values by the model with experimental data by Buscall et al., it is expected that (1)the value of $D$ increases with increasing $\phi_{0}$ and (2) the value of $D$ increases after prolonged shearing. Other researchers reported similar results like (1) by experiment $^{6)}$ and Brownian dynamics simulation ${ }^{7 /}$. 
a) $\mathrm{d}_{0}=0.488 \mu \mathrm{m}$

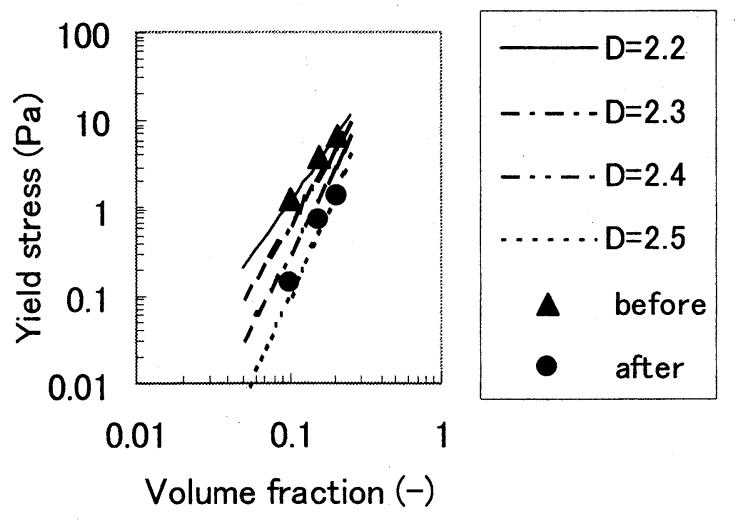

c) $d_{0}=2.62 \mu \mathrm{m}$

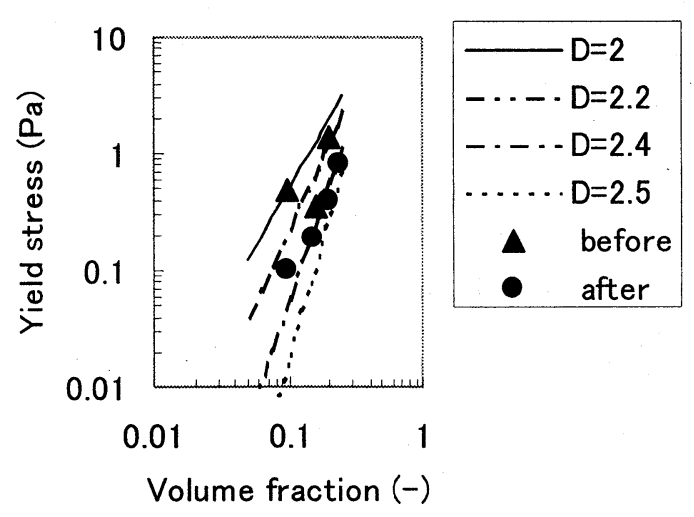

b) $d_{0}=0.966 \mu \mathrm{m}$

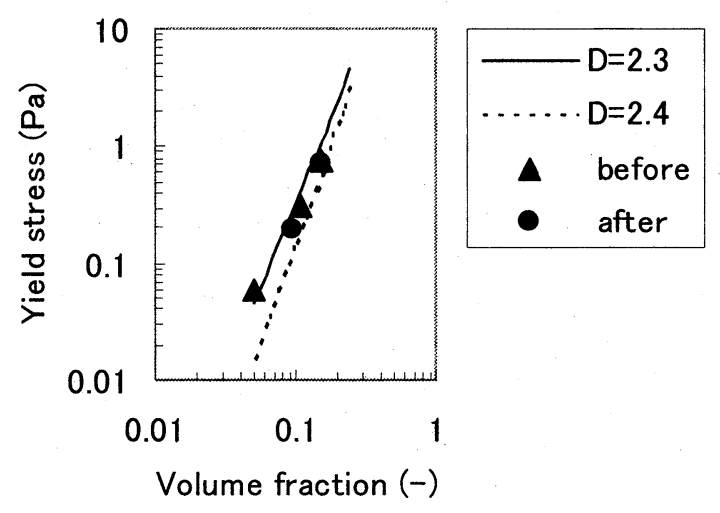

d) $\mathrm{d}_{0}=3.4 \mu \mathrm{m}$

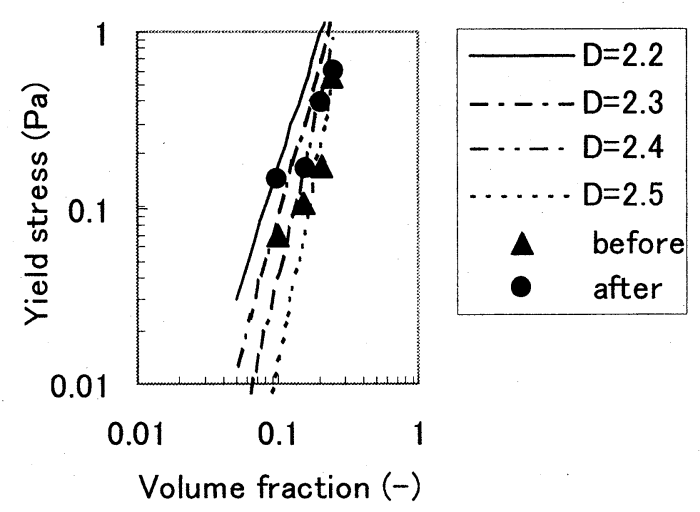

Fig. 3 Yield stress vs. volume fraction. Sy mbols and lines denote experimental data and evaluated values, respectively.

With regard to (2), it is known that flocs in sheared dilute suspensions have compact structure ${ }^{8,9)}$ although the effect of shear on floc structure is not reported for concentrated suspensions. These consistencies between previous studies and the present analysis validate the present model. But, further studies are needed because the reason why flocs become compact is still unclear.

\section{CONCLUSIONS}

A model of yield stress of coagulated suspensions is proposed. Evaluated values of yield stress by the model showed good agreement with the experimental results by Buscall et al. by supposing that flocs become compact in higher volume fractions and after prolonged shearing.

\section{ACKNOWLEDGMENTS}

The authors are thankful to Dr. Katsuya Nakaishi (Ibaraki University) and Dr. Kazumi Miyahara (Kyushu University) for their helpful comments and discussion. This work is financially supported by Grant-in-Aid for Scientific Research for JSPS research fellow.

\section{REFERENCES}

1) Ohgaki, S. and Matsuo, T.: Kounoudokendakueki no ryuudoutokusei ni tsuite, Doboku Gakkai Ronbun Houkokushu, Vol. 210, pp. 21-32, 1973, in Japanese.

2) Okuda, S.: Kancho kasen ni okeru taiseki kankyo, Kasen Kancho Iki, Saijo, Y. and Okuda, S. eds., Nagoya Daigaku Shuppan Kai, pp. 85-105, 1997, in Japanese.

3) van Kessel, T. and Blom, C.: Rheology of cohesive sediments: comparison between a natural and an artificial mud, J. Hydraulic Res., Vol. 36, pp. 591-612, 1998.

4) Kobayashi, M., Adachi, Y. and Ooi, S.: On the steady shear viscosity of coagulated suspensions, NIHON REOROJI GAKKAISHI, Vol. 28, pp. 143-144, 2000.

5) Buscall, R., Mills, P.D.A. and Yates, G.E.: Viscoelastic 
properties of strongly flocculated polystyrene latex dispersions, Colloids and Surfaces, Vol. 18, pp. 341-358, 1986.

6) Bremer, L.G.B.: Fractal aggregation in relation to formation and properties of particle gels, Thesis, Wageningen Agricultural University, 1992.

7) Mellema, M., van Opheusden, J.H.J and van Vliet, T.: Relating colloidal particle interactions to gel structure using Brownian dynamics simulations and the Fuchs stability ratio, J Chem. Phys., Vol. 111, pp. 6129-6135, 1999.
8) Hoekstra, L.L., Vreeker, R. and Agterof, W.G.M, T.: Aggregation of nickel hydxycarbonate studied by light scattering, J Colloid Interface Sci., Vol. 151, pp. 17-25, 1992.

9) Adachi, Y., Kobayashi, M. and Yanagibashi, T. in revision.

10) Mori, Y. and Ototake, N.: Kendakueki no nendo ni tsuite, Kagaku Kogaku, Vol. 20, pp. 488-494, 1956, In Japanese with English Abstract.

(Received October 1, 2001) 